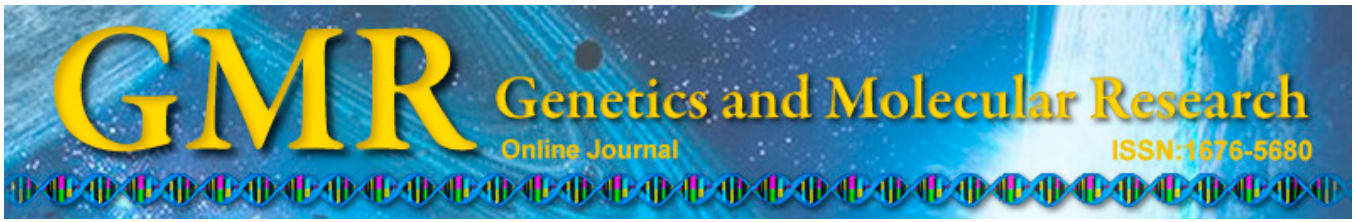

\title{
Pre-B-cell colony-enhancing factor is markedly elevated in childhood hemophagocytic lymphohistiocytosis
}

\author{
Z.Y. Ga0 ${ }^{1,2 *}$, X.Y. Li ${ }^{1,3 *}$, V. Bhandari' ${ }^{1}$ L.D. Li $^{1,2}$ and D. $\operatorname{Lan}^{1,2}$ \\ ${ }^{1}$ Department of Pediatrics, \\ The First Affiliated Hospital of Guang Xi Medical University, \\ Nanning, Guang Xi, China \\ ${ }^{2}$ National Center for International Research of Biological Targeting Diagnosis \\ and Therapy, Guang Xi Medical University, Nanning, Guang Xi, China \\ ${ }^{3}$ Present address: Department of Pediatrics, \\ The People's Hospital of Guangxi Zhuang Autonomous Region, \\ Nanning, Guang Xi, China \\ *These authors contributed equally to this study. \\ Corresponding author: D. Lan \\ E-mail: landan_ld@163.com
}

Genet. Mol. Res. 14 (2): 5287-5295 (2015)

Received September 16, 2014

Accepted January 19, 2015

Published May 18, 2015

DOI http://dx.doi.org/10.4238/2015.May.18.21

\begin{abstract}
Hemophagocytic lymphohistiocytosis (HLH) is a life-threatening syndrome involving a final common pathway of hypercytokinemia, in which tumor necrosis factor (TNF)- $\alpha$, interferon (IFN)- $\gamma$, and soluble interleukin 2-receptor-alpha (sIL-2R $\alpha$ ) are the key cytokines. Pre-B-cell colony-enhancing factor (PBEF) is an inflammatory cytokine involved in several inflammatory diseases. However, its role in HLH is unknown. In this study, we examined the role of PBEF in HLH. Plasma was collected from 22 children with HLH and 14 healthy children. The concentrations of plasma PBEF, TNF- $\alpha$, IFN- $\gamma$, and sIL$2 \mathrm{R} \alpha$ were determined using an enzyme-linked immunosorbent assay. All clinical data were derived from medical records. In the acute phase,
\end{abstract}


children with HLH had much higher PBEF, TNF- $\alpha$, IFN- $\gamma$, and sIL$2 \mathrm{R} \alpha$ levels than did healthy children $(\mathrm{P}<0.05)$. After treatment, 13 HLH children improved and PBEF, TNF- $\alpha$, and IFN- $\gamma$ levels decreased to normal levels $(\mathrm{P}<0.05)$; sIL-2R $\alpha$ levels also decreased $(\mathrm{P}<0.05)$, but remained above the normal level $(\mathrm{P}<0.05)$. Two patients were lost to follow-up, while 7 patients showed a bad response to therapy and eventually died, showing high PBEF levels above those of the survivors $(\mathrm{P}<0.01)$. PBEF level was significantly positively correlated with TNF- $\alpha$, IFN- $\gamma$, sIL-2R $\alpha$, serum ferritin, and triglycerides (all $\mathrm{P}<0.05$ ), and was negatively correlated with fibrin $(\mathrm{P}<0.05)$. PBEF appears to be involved in the inflammatory process of HLH, and elevated PBEF is related to disease activity. We are currently evaluating the role of PBEF as a marker for the diagnosis and management of patients.

Key words: Childhood; Cytokine; Hemophagocytic lymphohistiocytosis; Inflammatory process; Pre-B-cell colony-enhancing factor

\section{INTRODUCTION}

Human pre-B-cell colony-enhancing factor (PBEF) was first identified as a cytokine that acted synergistically with interleukin (IL)-7 and stem cell factor to stimulate early stage B cell formation (Samal et al., 1994). Several important regulatory elements for cytokines were identified in the sequence of the PBEF gene. However, Rongvaux et al. (2002) argued that PBEF is not a cytokine, as it did not contain known secretion sequences. Thus, whether PBEF is a cytokine remains unclear. However, numerous studies have indicated that PBEF acts as a cytokine and is implicated in several inflammatory diseases, including acute lung injury, clinical sepsis, and rheumatoid arthritis, among others (Jia et al., 2004; Liu et al., 2009; Senolt et al., 2011). PBEF has been found to upregulate a variety of inflammatory cytokines, including tumor necrosis factor (TNF)- $\alpha$, IL-1 $\beta$, IL-6, transforming growth factor- $\beta 1$, IL-16, and C-C chemokine receptor type 3 (Moschen et al., 2007; Song et al., 2008; Liu et al., 2009). At high concentrations, PBEF can also cause the production of some anti-inflammatory cytokines, such as IL-2-receptor-alpha (IL-2R $\alpha$ ) and IL-10 (Moschen et al., 2007). Additionally, PBEF was widely induced by TNF- $\alpha$, interferon (IFN)- $\gamma$, IL-1 $\beta$, IL-6, granulocyte macrophage colony-stimulating factor and other cytokines in neutrophils, monocytes, or macrophages (Jia et al., 2004; Nowell et al., 2006). Inhibition of PBEF significantly decreases the secretion of TNF- $\alpha$ (Evans et al., 2011). PBEF was shown to have an anti-apoptosis effect in macrophages during stress via the signal transducer and activator of transcription 3 survival pathway (Li et al., 2008). It also prevents the apoptosis of neutrophils by caspase- 3 and caspase- 8 during clinical sepsis (Jia et al., 2004).

Hemophagocytic lymphohistiocytosis (HLH), a potentially fatal syndrome that occurs regardless of age, is classified into genetic and acquired forms. The exact mechanism is relatively unclear for the latter form. Acquired HLH has been associated with numerous diseases, including infections and rheumatologic disease. The key pathophysiology for HLH is hyperactivation and hyperproliferation of cytotoxic $\mathrm{T}$ cells and macrophages, as well as uncontrolled inflammatory cytokine production, which can result in end-organ damage and even death (Tang and Xu, 2011). Most patients with HLH show elevated serum levels of 
soluble IL-2R (sIL-2R), TNF- $\alpha$, and IFN- $\gamma$ (Osugi et al., 1997). The main symptoms of HLH include persistent fever, pancytopenia, hepatosplenomegaly, and hemophagocytosis (Tang and $\mathrm{Xu}, 2011)$. Because most clinical features of HLH are nonspecific, establishing a diagnosis of HLH is time-consuming. The median time from the onset of the first signs of disease to diagnosis of HLH was 3.5 months in one study (Tang and $\mathrm{Xu}, 2011$ ). Because of this delayed recognition, the mortality of HLH was once as high as 95\% (Tothova and Berliner, 2014). Currently, clinicians are seeking innovative methods for early diagnosis. However, there have been no studies to examine the role of PBEF in HLH.

PBEF has been identified to react with several cytokines involved in HLH (Osugi et al., 1997); thus, we hypothesized that PBEF is involved in HLH, and understanding PBEF may be helpful for recognizing HLH. We therefore investigated the potential role of PBEF in childhood HLH.

\section{MATERIAL AND METHODS}

\section{Methods}

\section{Affected and control subjects}

This study was performed at the Department of Pediatrics, The First Affiliated Hospital of Guangxi Medical University, Nanning, Guang Xi, China. From May 2012 to February 2014, 22 children with HLH [male, 20; female, 2; median age, 1.6 years (1.9-3.3 years)] were included. Their clinical features are detailed in Table 1 (data were obtained before patients received therapy against HLH). Subjects with hematological malignancy-associated HLH were excluded. Fourteen age-matched healthy children who visited the hospital for a health examination were enrolled as the control group. No subjects in the control group were taking immunosuppressive drugs at the time of the visit. All clinical data were derived from medical records. Diagnosis and treatment of HLH were according to the "HLH-2004: Diagnostic and therapeutic guidelines for hemophagocytic lymphohistiocytosis" protocol (Henter et al., 2007). Non-survivors were defined as patients who died during the follow-up, whereas survivors were defined as patients who survived until the end of the study. We obtained informed consent from the guardians of all participants. Approval for this study was obtained from the Medical Ethics Committee at the First Affiliated Hospital of Guangxi Medical University.

\section{Specimen collection and measurement of plasma PBEF, TNF- $\alpha, I F N-\gamma$, and sIL-2Ra concentrations}

Plasma was collected and stored at $-80^{\circ} \mathrm{C}$. We collected plasma from children with HLH at 4 time points, including pre-therapy, 1 week after therapy, 2 weeks after therapy, or on the day of death. Because 2 patients were lost to follow-up after chemotherapy, we failed to collect their plasma after treatment. The concentrations of plasma PBEF, TNF- $\alpha$, IFN- $\gamma$, and sIL-2R $\alpha$ were determined using an enzyme-linked immunosorbent assay (ELISA). ELISA kits were used for the ELISA assay (PBEF ELISA kit was obtained from Phoenix Pharmaceuticals, Inc., Burlingame, CA, USA; TNF- $\alpha$, IFN- $\gamma$, and sIL-2R $\alpha$ ELISA kits were obtained from ImmunoWay Biotechnology Company, Newark, DE, USA). Optical density was determined at $450 \mathrm{~nm}$ using a Multiskan Spectrum (Thermo Fisher Scientific, Waltham, MA, USA) and the results were calculated using the SoftMaxPro6.3 software. 


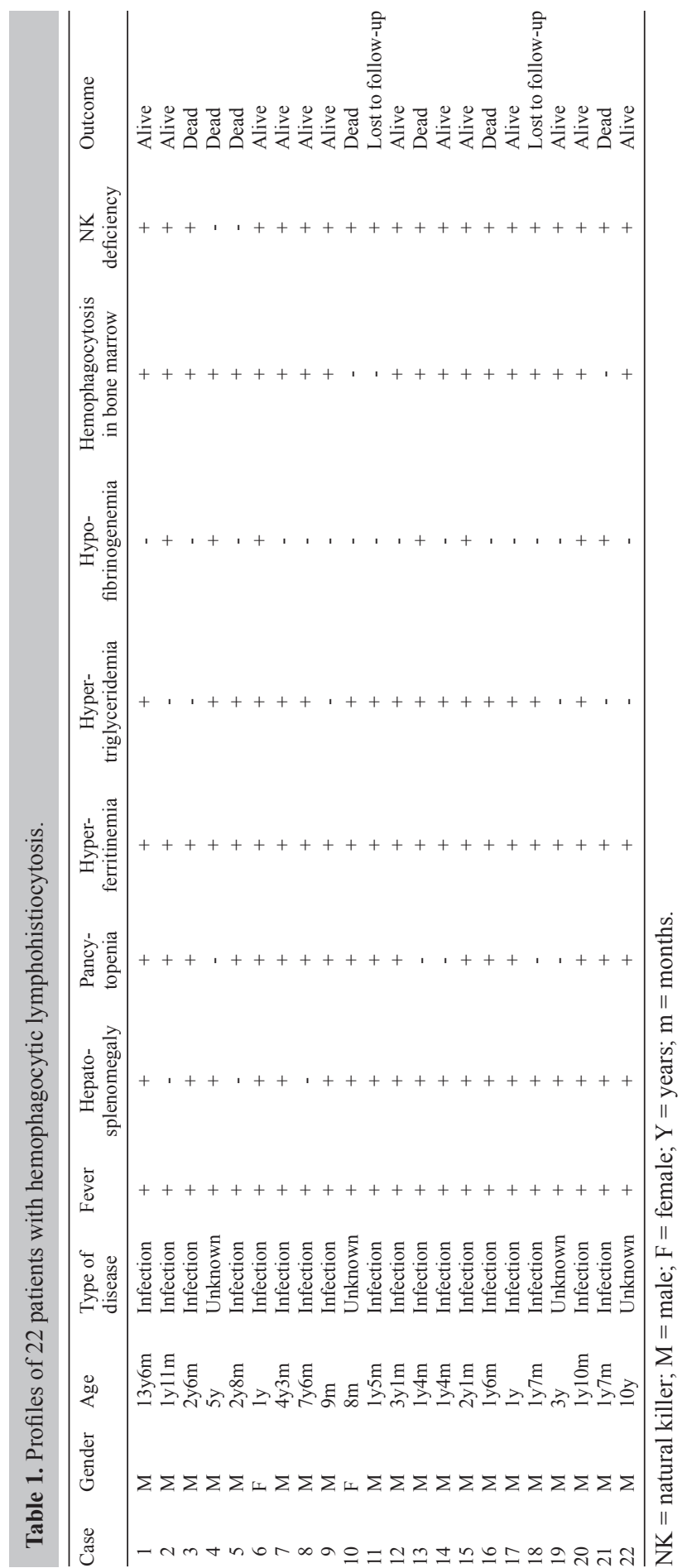




\section{Statistical analysis}

The statistical package SPSS V. 10.0 was used to analyze the data (SPSS, Inc., Chicago, IL, USA). Continuous variables are reported as the median value with interquartile range (2575th percentile), or as means \pm standard deviation. Wilcoxon rank-sum test was performed to examine the difference in PBEF, TNF- $\alpha$, IFN- $\gamma$, and sIL-2R $\alpha$ concentrations between groups. The Spearman rank correlation test was used to analyze the correlations between PBEF and other cytokines as well as laboratory findings. Statistical significance was set at $\alpha=0.05$ (two-tailed).

\section{RESULTS}

\section{Higher PBEF levels in HLH patients than in healthy controls}

In the acute phase of $\mathrm{HLH}$, the median concentration of PBEF before chemotherapy was 33.78 (10.28-63.40) ng/mL, while the PBEF level in healthy children was only 5.37 (4.84-6.38) $\mathrm{ng} / \mathrm{mL}$. Figure $1 \mathrm{~A}$ shows the comparison of plasma PBEF concentrations between groups. Before chemotherapy, children with HLH had much higher PBEF levels than healthy children $(\mathrm{P}=0.003)$.
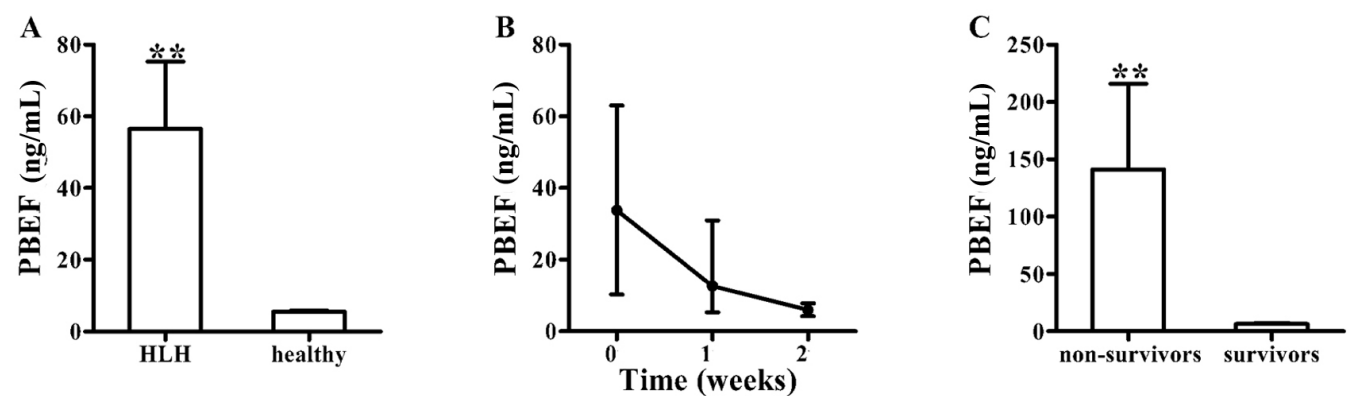

Figure 1. Plasma PBEF levels in the studied individuals. We determined the concentrations of plasma PBEF using ELISA. Data represent median (IQR) values. A. Comparison of PBEF levels between children with HLH (in acute phase) and healthy individuals. PBEF was markedly elevated in HLH subjects. ${ }^{*} * \mathrm{P}<0.01$. B. Time course of PBEF levels in children with HLH who showed good curative effect. PBEF levels decreased with disease improvement. 0: pre-therapy; 1: 1 week after therapy; 2: 2 weeks after therapy. C. Comparison of plasma PBEF levels between nonsurvivors and survivors. After chemotherapy, non-survivors showed much higher PBEF levels than the survivors. $* * \mathrm{P}<0.01$.

\section{PBEF levels were correlated with the severity of HLH}

As described above, children with HLH in the acute phase showed high PBEF levels before treatment. After treatment against HLH, 13 patients showed a good therapeutic effect and were in stable condition. Seven patients showed further deterioration until they eventually died. Two patients were lost to follow-up.

Figure 1B shows the decreasing trend in PBEF levels in children with HLH showing an improved condition after treatment. After 1 week of chemotherapy, the PBEF level slightly decreased to 12.63 (5.27-30.94) $\mathrm{ng} / \mathrm{mL}(\mathrm{P}>0.05)$. After 2 weeks of treatment, the PBEF level significantly decreased to $5.87(4.14-7.75) \mathrm{ng} / \mathrm{mL}(\mathrm{P}=0.001)$, which was comparable to that in healthy children $(\mathrm{P}=0.550)$.

After chemotherapy, PBEF levels in non-survivors were significantly higher than 
those observed in survivors [101.551 (20.168-114.831) vs 6.34 (4.472-7.631) ng/mL; P < 0.001 , Figure 1C].

\section{PBEF was significantly correlated with laboratory findings}

High levels of serum ferritin and triglycerides, as well as low fibrinogen are widely used markers for the diagnosis of HLH. We also investigated the correlation between PBEF and these laboratory findings. PBEF level showed a significant positive correlation with serum ferritin $(\mathrm{r}=0.627, \mathrm{P}<0.05)$ and triglyceride levels $(\mathrm{r}=0.633, \mathrm{P}<0.05)$, and negative correlation with fibrin $(\mathrm{r}=-0.521, \mathrm{P}<0.05)$.

\section{TNF- $\alpha$, IFN- $\gamma$, and sIL-2R $\alpha$ levels were positively correlated with PBEF, while their evolution during the clinical course of HLH was identical with PBEF}

TNF- $\alpha$, IFN- $\gamma$, and IL-2R are important cytokines in HLH. In this study, we found a positive correlation between PBEF and TNF- $\alpha(\mathrm{r}=0.460, \mathrm{P}<0.05)$, IFN- $\gamma(\mathrm{r}=0.512, \mathrm{P}<$ $0.05)$, and sIL-2R $\alpha(\mathrm{r}=0.461, \mathrm{P}<0.05)$.

Moreover, changes in TNF- $\alpha$, IFN- $\gamma$, and sIL-2R $\alpha$ levels over the clinical course of HLH were identical with that of PBEF. The concentrations of each cytokine for each group are shown in Table 2. As shown in Figure 2, patients in the acute phase of HLH showed higher levels of plasma TNF- $\alpha$, IFN- $\gamma$, and sIL-2R $\alpha$ than did healthy children. After treatment for 2 weeks, some patients improved, and the concentrations of all cytokines decreased with clinical improvement. The TNF- $\alpha$ and IFN- $\gamma$ concentrations decreased to normal levels $(\mathrm{P}<0.05$; Figure 2A and $\mathrm{B})$; sIL-2R $\alpha$ levels were also significantly decreased $(\mathrm{P}<0.05)$, but remained above the normal level $(\mathrm{P}<0.05$; Figure $2 \mathrm{C})$.

\begin{tabular}{|c|c|c|c|}
\hline & \multicolumn{2}{|c|}{ HLH children } & \multirow[t]{2}{*}{ Healthy children } \\
\hline & Pre-therapy & 2 weeks after therapy & \\
\hline $\mathrm{TNF}-\alpha(\mathrm{pg} / \mathrm{mL})$ & $395.93 \pm 158.51$ & $236.44 \pm 139.29$ & $184.08 \pm 114.80$ \\
\hline IFN- $\gamma(\mathrm{pg} / \mathrm{mL})$ & $1544.80 \pm 696.29$ & $659.83 \pm 378.56$ & $553.73 \pm 351.44$ \\
\hline $\mathrm{sIL}-2 \mathrm{R} \alpha(\mathrm{pg} / \mathrm{mL})$ & $2493.89(2168.74-3819.64)$ & $1557.10(1144.26-2234.61)$ & 993.09 (800.81-1074.48) \\
\hline
\end{tabular}
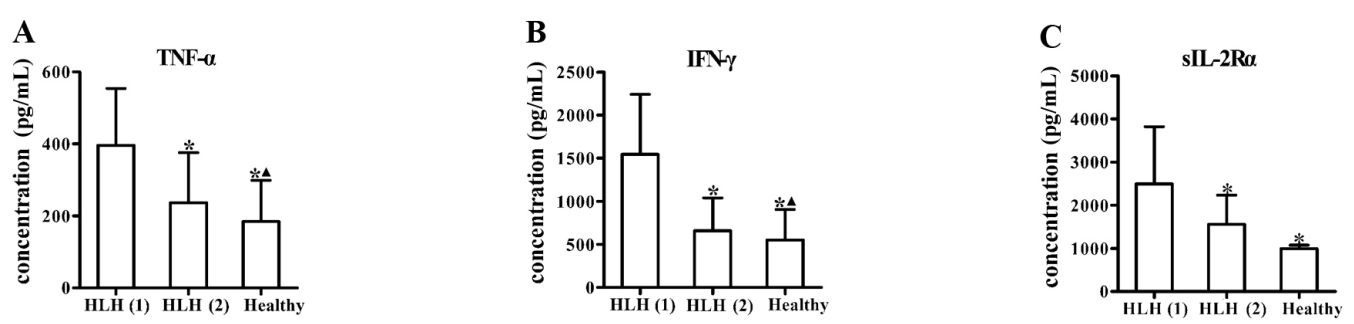

Figure 2. Comparison of plasma TNF- $\alpha$, IFN- $\gamma$, and sIL-2R $\alpha$ levels between groups. HLH (1): children in acute phase of HLH; HLH (2): children with HLH who showed improvement after treatment. Similar to PBEF, TNF- $\alpha$, IFN- $\gamma$, and sIL-2R $\alpha$ levels were also correlated with the disease activity of HLH. During the acute phase of HLH, patients showed much higher levels of these cytokines than did healthy individuals. After effective treatment, TNF- $\alpha$ and IFN- $\gamma$ levels had decreased to normal levels (A and B); sIL-2R $\alpha$ levels had also decreased, but were still above the normal level (C). * $\mathrm{P}<0.05$ compared with HLH (1) group. ${ }^{\star} \mathrm{P}<0.05$ compared with HLH (2) group. 


\section{DISCUSSION}

PBEF has been found to be associated with several inflammatory diseases, including acute lung injury, clinical sepsis, and rheumatoid arthritis, among others (Jia et al., 2004; Liu et al., 2009; Senolt et al., 2011). However, this is the first study to examine PBEF in childhood HLH. Notably, children with HLH showed markedly elevated PBEF levels during the acute phase. Furthermore, PBEF level was well-correlated with disease activity of HLH, and decreasing PBEF levels were observed with disease improvement. After chemotherapy, we also observed that non-survivors showed much higher PBEF levels than survivors. These results indicate that PBEF level reflects the inflammatory reaction status. PBEF was correlated with the severity of HLH, and a higher PBEF level may be associated with worse clinical prognosis.

According to the current diagnostic criteria, laboratory findings are helpful for diagnosing HLH. High levels of ferritin and triglycerides, as well as low fibrinogen, are considered to indicate HLH. In the present study, PBEF was significantly correlated with these laboratory findings. Therefore, in combination with known biomarkers, the determination of PBEF may be helpful for recognizing HLH.

These results may be because of the involvement of PBEF in the inflammatory process of HLH. PBEF may be involved in cytokine upregulation in HLH. HLH is a syndrome characterized by severe hyperinflammation induced by an exaggerated but ineffective immune reaction. Cytotoxic T-cells that undergo prolonged activation continue to proliferate and to produce large amounts of IFN $-\gamma$, inducing uncontrolled macrophage activation, and then cause secretion of large amounts of IL-12 and other cytokines such as IFN- $\gamma$ and TNF- $\alpha$ (Lykens et al., 2011). Activated lymphocytes release large amounts of sIL-2R $\alpha$. IFN- $\gamma$ and TNF- $\alpha$ are active during the acute phase of $\mathrm{HLH}$, and decrease to normal levels after remission (Osugi et al., 1997). The expression of PBEF can be induced by TNF- $\alpha$ and IFN- $\gamma$ (Patrone et al., 2002; Jia et al., 2004; Moschen et al., 2007). In order to test this hypothesis, we analyzed plasma TNF- $\alpha$, IFN- $\gamma$, and sIL-2R $\alpha$ concentrations in children with HLH and in healthy children. We found that TNF- $\alpha$, IFN- $\gamma$, and sIL-2R $\alpha$ levels were well-correlated with disease activity; during the acute phase, they were much higher than those in healthy individuals, and declined with the clinical improvement after effective treatment. Changes in these cytokines during the clinical course of HLH were identical to those for PBEF. Moreover, a positive correlation was observed between PBEF and IFN- $\gamma$, TNF- $\alpha$, and sIL-2R $\alpha$.

These findings support the hypothesis that PBEF participates in the cytokine induction in HLH. However, the causal relationship between PBEF and HLH requires further investigation. The difference between the genetic and acquired forms must also be identified. In recent years, PBEF has been confirmed to be a treatment target for some inflammatory diseases (Evans et al., 2011). The results of this study may lay the foundation for the development of novel PBEF-targeted protocols to inhibit the abnormal inflammatory response in HLH.

In addition, plasma PBEF level in children with HLH was much higher than in children with sepsis and acute lymphocytic leukemia (ALL). The expression of PBEF mRNA in ALL cells was below normal levels. Distinguishing between HLH and sepsis is difficult. ALL is one of the most common malignant diseases associated with HLH (Tothova and Berliner, 2014). Some clinical manifestations of HLH are similar to those of ALL. However, timely diagnosis and targeted therapy are key, life-saving measures for patients. This is a difficult challenge faced by clinicians. Perhaps the determination of PBEF could help clinicians determine a differential diagnosis of HLH and sepsis or ALL. 
Although most children with HLH in our cohort had the acquired form, because of the lack of HLH-related genetic testing results, we could not confirm the classification of children with HLH as having the acquired or genetic forms. Although this study is preliminary, we found that plasma PBEF was markedly elevated in childhood HLH. Additional studies are needed to confirm our present findings.

In conclusion, PBEF may be involved in the inflammatory process of HLH. An elevated PBEF level was observed in children with $\mathrm{HLH}$, which was highly positively correlated with disease activity. PBEF was correlated with the widely available biochemical markers for diagnosing HLH, as well as predominating cytokines involved in HLH. This may be useful in the diagnosis of HLH. Higher PBEF appears to be associated with a worse prognosis. We are currently further evaluating its role as a marker for the early diagnosis and management of patients.

\section{ACKNOWLEDGMENTS}

Research supported by the National Natural Science Foundation of China (grants \#81060045, \#81170482).

\section{REFERENCES}

Evans L, Williams AS, Hayes AJ, Jones SA, et al. (2011). Suppression of leukocyte infiltration and cartilage degradation by selective inhibition of pre-B cell colony-enhancing factor/visfatin/nicotinamide phosphoribosyltransferase: Apo866mediated therapy in human fibroblasts and murine collagen-induced arthritis. Arthritis Rheum. 63: 1866-1877.

Henter JI, Horne A, Aricó M, Egeler RM, et al. (2007). HLH-2004: Diagnostic and therapeutic guidelines for hemophagocytic lymphohistiocytosis. Pediatr. Blood Cancer 48: 124-131.

Jia SH, Li Y, Parodo J, Kapus A, et al. (2004). Pre-B cell colony-enhancing factor inhibits neutrophil apoptosis in experimental inflammation and clinical sepsis. J. Clin. Invest. 113: 1318-1327.

Li Y, Zhang Y, Dorweiler B, Cui D, et al. (2008). Extracellular Nampt promotes macrophage survival via a nonenzymatic interleukin-6/STAT3 signaling mechanism. J. Biol. Chem. 283: 34833-34843.

Liu P, Li H, Cepeda J, Zhang LQ, et al. (2009). Critical role of PBEF expression in pulmonary cell inflammation and permeability. Cell Biol. Int. 33: 19-30.

Lykens JE, Terrell CE, Zoller EE, Risma K, et al. (2011). Perforin is a critical physiologic regulator of T-cell activation. Blood 118: 618-626.

Moschen AR, Kaser A, Enrich B, Mosheimer B, et al. (2007). Visfatin, an adipocytokine with proinflammatory and immunomodulating properties. J. Immunol. 178: 1748-1758.

Nowell MA, Richards PJ, Fielding CA, Ognjanovic S, et al. (2006). Regulation of pre-B cell colony-enhancing factor by STAT3-dependent interleukin-6 transsignaling: implications in the pathogenesis of rheumatoid arthritis. Arthritis Rheum. 54: 2084-2095.

Osugi Y, Hara J, Tagawa S, Takai K, et al. (1997). Cytokine production regulating Th1 and Th2 cytokines in hemophagocytic lymphohistiocytosis. Blood 89: 4100-4103.

Patrone L, Damore MA, Lee MB, Malone CS, et al. (2002). Genes expressed during the IFN gamma-induced maturation of pre-B cells. Mol. Immunol. 38: 597-606.

Rongvaux A, Shea RJ, Mulks MH, Gigot D, et al. (2002). Pre-B-cell colony-enhancing factor, whose expression is upregulated in activated lymphocytes, is a nicotinamide phosphoribosyltransferase, a cytosolic enzyme involved in NAD biosynthesis. Eur. J. Immunol. 32: 3225-3234.

Samal B, Sun Y, Stearns G, Xie C, et al. (1994). Cloning and characterization of the cDNA encoding a novel human preB-cell colony-enhancing factor. Mol. Cell. Biol. 14: 1431-1437.

Senolt L, Kryštůfková O, Hulejová H, Kuklová M, et al. (2011). The level of serum visfatin (PBEF) is associated with total number of B cells in patients with rheumatoid arthritis and decreases following B cell depletion therapy. Cytokine 55: $116-121$ 
Song HK, Lee MH, Kim BK, Park YG, et al. (2008). Visfatin: a new player in mesangial cell physiology and diabetic nephropathy. Am. J. Physiol. Renal Physiol. 295: F1485-F1494.

Tang YM and Xu XJ (2011). Advances in hemophagocytic lymphohistiocytosis: pathogenesis, early diagnosis/differential diagnosis, and treatment. Sci. World J. 11: 697-708.

Tothova Z and Berliner N (2014). Hemophagocytic syndrome and critical illness new insights into diagnosis and management. J. Intensive Care Med. [Epub ahead of print]. 